NOTICE: This is the authors' version of a work that was accepted for publication in SAGE Methods Cases. Changes resulting from the publication process, such as peer review, editing, corrections, structural formatting, and other quality control mechanisms may not be reflected in this document. Changes may not have been made to this work since it was submitted to publication. A definitive version was subsequently published in SAGE Methods Cases. doi: $10.4135 / 9781526401083$ 


\title{
Case Title
}

Community-Based Participatory Research with LGBTQ Communities in Alabama and Mississippi

\section{Author Name(s)}

\author{
Kamden K. Strunk
}

Hannah Carson Baggett

Andrea Riemer

Raina Hafftka

\section{Author Affiliation \& Country of Affiliation}

Auburn University; Research Initiative on Social Justice and Equity; USA

Auburn University; USA

Auburn University; USA

Auburn University; USA

\section{Lead Author Email Address}

Email:kks0013@auburn.edu

\section{Contributor Biographies}

Kamden Strunk, Ph.D. is an Assistant Professor of Educational Research at Auburn University, and a director of the Research Initiative on Social Justice and Equity (RISE). His research focuses on equity and inclusion, particularly in higher education. His work focuses on LGBTQ students and their experiences in higher education. He is a quantitative methodologist, teaching courses in statistical analysis for educational research.

Hannah Baggett, Ph.D. is an Assistant Professor of Educational Research at Auburn University. Her primary line of inquiry integrates critical race theory with psychological research about teachers' beliefs to examine how teacher education prepares teachers to meet the needs of diverse learners. She also uses qualitative and participatory research methods to examine inequity in educational contexts and beyond. She teaches courses in qualitative research methods and basic methods for education research.

Andrea Riemer is a graduate student pursuing a Ph.D. in Educational Leadership at Auburn University, where she is also pursuing a graduate certificate in Program Evaluation. She also serves as a Graduate Research Assistant and Graduate Teaching Assistant. Her research interests include the experiences of boys in the primary and secondary school; especially the 
experiences of male students of color. Currently, she teaches at the elementary level in Auburn, Alabama.

Raina Hafftka a graduate student pursuing a Ph.D. in Counseling Psychology at Auburn University. She has experience in clinical and research positions. Her research involvement has spanned areas relating to LGBTQ individuals, disclosure in psychotherapy, evolutionary psychology, and education type differences relating to achievement.

\begin{abstract}
This case study is based on our work with community-based participatory research with LGBTQ communities in Alabama and Mississippi. We describe the process of conducting community-based participatory research in this population, with particular focus on some of the obstacles to success, and some of the strategies that were useful for our work. We discuss the Institutional Review Board process for this study, the process of identifying and recruiting community researchers, our process of providing research training, and the analytic process for this work. In particular, we describe the steps we took to present our study to the Institutional Review Board to ensure clarity and ethical compliance. We articulate our recruitment strategies, including coordination with community organizations, and the use of social media for recruiting. We describe the process we undertook to train community members in research ethics and interviewing skills. Finally, we describe the process we are involved in for data analysis that includes community collaboration. Our goal is to provide a case study which can be useful for those considering a community-involved approach to understanding marginalized populations.
\end{abstract}

\title{
Learning Outcomes
}

1. Students will understand and be able to describe the concepts of community-involved research, and community-based participatory research.

2. Students will be able to apply concepts from community-based participatory research to understanding marginalized populations.

3. Students will be able to identify useful practices for community-involved research, and apply those practices to their own research work.

4. Students will be able to create a plan for community involvement in their own ongoing, thesis, or dissertation research.

\section{Project Overview and Context}

\section{Case Study}

In this case study, we describe a community-involved research project with the goal of understanding the needs and experiences of LGBTQ people in Alabama and Mississippi, especially LGBTQ people of color. This study, which was funded by an internal grant from Auburn University's Outreach office, has involved multiple sessions with community members across both states, collaborations with multiple community organizations, and collection of data by community members. Here, we summarize the theory and practice of 
community-based participatory research (CBPR), our project with LGBTQ people in the U.S. South, the challenges in designing CBPR research, particularly within funding and institutional constraints, and the lessons we have learned from this project.

Community-Based Participatory Research. The methodology used in this study was community-based participatory research (CBPR). CBPR emerged from public health research where it has been useful in the understanding of and intervention on public health issues. There are many variations to CBPR methodology, responsive to various needs of the researchers, participants, communities, and problems. For example, Participatory Action Research (PAR) and Youth Participatory Action Research (YPAR) are both similar methods for integrating community engagement, research practices, and stakeholders to identify and ameliorate problems in a progressive, activist manner (Johnson, 2017; Ozer, 2016; Whyte, 1989). The commonality among various participatory research approaches is that each promotes a shift in power or control from researchers to community members.

Community-Based Participatory Research methods (CBPR) are particularly beneficial when researching and working alongside marginalized communities. CBPR techniques create opportunities for community members themselves to participate in the development of the research project, to identify the most important needs of their community, to conduct the studies and assessments, and to solicit the resources necessary to change policies or resolve problems. The utility of the CBPR model is that it is collaborative and promotes empowerment of marginalized communities. However, that same empowerment and collaboration presents challenges to the implementation of CBPR methods, some of which are particularly salient to work with LGBTQ populations in the U.S. South.

LGBTQ Communities in Alabama and Mississippi. The Williams Institute (2016) estimates, based on census data, that at least 164,000 LGBTQ people live in Alabama and Mississippi. While attitudes toward LGBTQ people have been improving nationwide, Mississippi and Alabama rate consistently lower on acceptance of LGBTQ people (Williams Institute, 2016). Yet, over 35\% of LGBTQ people in the U.S. live in the Deep South - the largest proportional representation of any U.S. geographical region (Mallory, Flores, \& Sears, 2016). Bias and discrimination are real and ongoing problems for LGBTQ people in the Deep South. In Alabama, for example, $81 \%$ of all residents believe that LGBTQ people are discriminated against (Mallory, Flores, \& Sears, 2016). In a 2014 large-scale survey on LGBTQ people in the Deep South, 38\% had experienced harassment at work, $48 \%$ have been harassed in public accommodations, $46 \%$ have been harassed at school, and $47 \%$ do not have access to LGBTQ-friendly healthcare services (Human Rights Campaign, n.d.). It is clear, then, that while the LGBTQ community in Alabama and Mississippi is relatively large, it is also a marginalized and potentially vulnerable community.

Prior Research and Impetus for the Study. Research regarding LGBTQ people in the U.S. South is sparse, and even less common regarding LGBTQ people of color in the South. Among those studies, many focus on sexually transmitted infections and HIV/AIDS (Berg \& Ross, 2014; MacCarthy, et al., 2015; Slater, et al., 2015). Others relate more broadly to health among LGBTQ people in the South (Austin \& Irwin, 2010a; Austin \& Irwin, 2010b). A few have included research questions related to mental health (Craig \& Keane, 2014; Shelton \& Delgado-Romero, 2011). Very few relate to issues of experiences of LGBTQ people in the South, and are almost universally focused on experiences of bias and discrimination (Baunach, Burgess, \& Muse, 2010; Geter, et al., 2016). The existing research, with few exceptions, focuses on White LGBTQ people, as well. The end result is that little is 
known about the experiences of LGBTQ people in the South, and even less is known about the experiences of LGTBQ people of color in the South. Community-based research offers a way to reach this marginalized community, to understand their experiences and needs from a strength-based perspective, and to meaningfully involve communities in creating change.

\section{Designing Research for Community Involvement}

Typical Steps in Community-Based Participatory Research. The most typical approaches to CBPR follow a similar pattern (e.g., Hacker, 2013):

1. Identification of the community

2. Collaboration with the community to define research questions

3. Working with the community to determine research methods

4. Community members aid in or conduct data collection

5. Researchers work with community members in analysis

6. Researchers return the findings to the community

Similarly, Johnson (2017) identifies three crucial elements in community-based research:

1. Collaborative-Community members are viewed as equal partners in the research, opportunities for discussion and dialogue are provided, and leadership is shared with community members.

2. Critical-CBPR work should challenge the dominant narratives, provide alternative voices, and connect local conditions to larger societal systems.

3. Transformative - Researchers in CBPR work should be oriented toward taking action, should seek to change perspectives and practices, and should be a transformational learning and teaching experience.

These steps and guidelines are useful in conceptualizing how to do research with communities instead of doing research on communities. These strategies move researchers to think about communities as real partners in research and as capable of self-direction, rather than thinking about communities as objects of study. As we will explore, however, those guidelines and steps also present real challenges for researchers related to issues of community and to institutional structures.

Who Is the Community? An obvious, but difficult, first step in enacting CBPR methods is to define and reach a 'community'. In some cases, this is simple. Public health researchers have defined towns or cities as communities for the purposes of health interventions. These geographically bound communities are easier to define, and perhaps easier to reach, than communities defined by shared characteristics or goals.

For our purposes, we defined the 'community' as individuals who identified on the LGBTQ spectrum, and who lived in Alabama or Mississippi. This loose definition is in some sense reasonable. The words 'LGBTQ' and 'community' are so often linked, it is easy to forget that, particularly in the U.S. South and in rural areas, there can be a real lack of sensed community among LGBTQ people. LGBTQ communities in more urban and more progressive areas tend to form around LGBTQ community centers, LGBTQ nightlife locations, even LGBTQ 'districts' that exist in some areas with high concentrations of LGBTQ residents and businesses. These districts exist in larger urban areas, like Atlanta, GA or Dallas, TX, but are absent in Alabama and Mississippi for the most part. In rural areas, and in large portions of the U.S. South, such spaces simply do not exist. From our observations in the present study, when such spaces do exist, the sense of community is enhanced. However, 
when visiting small towns and rural areas of Mississippi and Alabama, LGBTQ spaces are rare. How, then, do we define and reach the LGBTQ community?

For us, the answer has been reaching out through existing organizations and social media. We have identified and partnered with key community groups, including one national group and two local groups. Each of these organizations has some level of existing community, whether that means existing volunteers, followers on social media, or actual community groups. In addition to those existing networks, we sought to expand to more LGBTQ people through targeted social media advertisements. Interestingly, reaching those individuals through social media often resulted in them, in one way or another, connecting with partner organizations as well.

Means of Increasing Community Involvement. Having identified, though quite loosely, what we meant by the LGBTQ community in Alabama and Mississippi, and reaching out to that community through existing organizations and social media, we had to determine ways to promote meaningful community involvement. Because of challenges to full collaboration arising from institutional structures, which we describe more fully later in this case, community involvement upfront was limited. We consulted with several organizations and their leaders, but the essential takeaway from those conversations was that data are lacking, and especially so for LGBTQ people of color. We arrived at the conclusion, from those conversations, that data were needed. We also decided on a community-involved research strategy to overcome some of the challenges with reaching LGBTQ people, especially LGBTQ people of color, as identified by prior researchers.

We have continued to coordinate our efforts with organizations as we have moved through this research process. However, it has been a decidedly researcher-driven process. This is counter to the core ideas of CBPR, but has occurred partially due to institutional factors and partially due to community factors. Our approach has been, given the fact that our process was ultimately researcher-driven, to increase community involvement in data collection, analysis, and interpretation. We have done this through training sessions for community researchers, providing equipment, supplies, and materials for community researchers to conduct qualitative interviews, and by scheduling conference calls and webinars to discuss results and interpretation.

\section{Challenges in Community-Based Participatory Research}

Challenges to Collaborative Research Design in CBPR. When communities lack affiliation and feel disempowered, collaborative design is difficult. We found that for LGBTQ people in Alabama and Mississippi, there was a real sense of disempowerment. The states' governments have been consistently hostile to LGBTQ citizens, and the community members we worked with reported constant experiences of marginalization and bias. Perhaps related to those experiences, the number of community members interested and willing to become community researchers was smaller. Adding to this problem is the lack of 'out-ness' among LGBTQ people of color. Conversations with community groups and community members revealed that many LGBTQ people of color were unwilling to be open about their sexual and/or gender identity due to fear of reprisal, experiences of stigma, or anxiety about how that openness might affect personal or professional relationships.

Additionally, identifying stakeholders to assist in design can be difficult. One example is our work with the regional office of a national LGBTQ organization. That 
organization was helpful in identifying community members we might work with, and provided us with meeting space free of charge. However, our work with that organization was not entirely accepted by other community organizations. In conversations with other local and regional organizations, they questioned our work with the national group and what it might reveal about our intentions. Beyond these complications among organizations, we also wondered how representative the membership of any LGBTQ organization operating in the South might be. Most LGBTQ people in the South are not involved with any community organizations. Could we trust that those who were members were in some way typical of the population?

Tensions between Funding Models, Institutional Approvals, and CBPR. We recognized early in designing a study of the needs and experiences of LGBTQ people in Alabama and Mississippi that we would need funding to successfully carry out such a study. Not only would the study require our research team to travel extensively, but we would need help from graduate research assistants (who also would need to travel extensively). In addition, we would need to provide appropriate resources to community researchers, like informational packets, readings, digital voice recorders, and money for participation incentives. Our institution, Auburn University, thankfully had a friendly internal funding mechanism for projects that involve outreach or community involvement. However, preparing the application for that funding presented a major challenge for our efforts at collaboration and allowing a design to emerge from community interactions, both of which are consistent with CBPR as it is defined in the research literature (Hacker, 2010; Johnson, 2016). Applying for that funding required us to design the study completely, including budget and data collection materials, to list sites we would visit, and essentially detail the entire research process. Thus, doing so collaboratively proved challenging.

We worked with a representative of one community organization, and sought a letter of support for our funding proposal from that organization as well. However, we quickly realized that the organization may have had a false sense of how likely we were to be funded. The organization representative began working to identify dates for us to visit and spaces we could use to meet. While the representative's enthusiasm was energizing and welcomed, we came to realize it also suggested if we were not funded, it would likely lead to disappointment and damaged relationships with that organization. As a result, we decided not to include any other organizations in the initial planning of our funding proposal. In hindsight, we would have worked harder to find ways to temper expectations while also encouraging deeper collaborations.

Our funding proposal was approved, but required (as many do) IRB approval before spending the funds. A further challenge to collaborative design came when we applied for Institutional Review Board (IRB) approval of our work. Recruiting community members to work on the project, we learned, would be thought of by the IRB as recruiting for human subjects research. As a result, we needed IRB approval before recruiting community members into the project. That meant we had to specify even more detail in our plans than we had for the funding proposal, and had to do so without meaningful involvement of the community members with whom we would eventually work. There are ways around this problem: We could have submitted an initial IRB protocol to recruit community members to discuss the research design, then submitted modifications along the way as the research design was solidified and created collaboratively with community members. For our purposes, our funding window was one year. Our initial IRB review took about 5 weeks, which is typical at our institution. To request modifications along the way while working 
with community researchers would likely have required at least three and as many as six additional reviews of modifications, leading to a total time in IRB review of between 15 and 30 weeks (over half of our funding window). These institutional and financial realities led us to minimal community involvement in the planning of the project, defining of research questions, and selection of data collection methods.

\section{Specific Challenges in LGBTQ Community-Based Participatory Research}

LGBTQ Communities in the South. As discussed previously, our 'community' in this case was difficult to define due to our community researchers' lack of affiliation with a discrete community, or existing connections with other LGTBQ people in their areas. In this case, some of our community researchers reported being drawn to participate because they felt extremely isolated in their own cities and towns, and were looking for opportunities to further connect with other LGBTQ people in the area. We anticipated that it would be particularly difficult for these researchers to recruit and interview other LGBTQ people in their areas since they already felt a lack of community. In these instances, we were grateful for the presence of our partnership organizations, as they provided these researchers with resources and literature regarding the area. We reflected as a research team that our sessions, even if they did not yield meaningful data collection, served an unanticipated purpose in providing a safe space for community members to connect.

Bias, Discrimination, and Stigma. The treatment of LGBTQ people in the South can create an atmosphere where being a community researcher is risky, as is being a participant. For example, some of our community researchers who had previously lived in places where they reported feeling comfortable being 'out' no longer felt that level of comfort in some regions in Alabama and Mississippi. Community researchers also reported workplace and hiring discrimination in certain areas. In our sessions, we discussed ways to mitigate 'outing' both their participants and themselves in instances when they felt unease at identifying themselves as LGBTQ, such as choosing pseudonyms and meeting for interviews at spaces that were perceived as 'safe'.

Multiple Marginalization. LGBTQ people are already marginalized, but in the South many are people of color, low-income, and have limited formal education. At the onset of the project, we had not anticipated what these factors might mean for interactions with university researchers. For example, in our initial session with researchers in Alabama, we, the university-affiliated researchers and graduate research assistants, introduced ourselves as such. That is, the primary way we identified ourselves, along with talking about more personal identity markers, was that of our university affiliation. Some of our community researchers had attended local universities or colleges, but others had not. As community researchers introduced themselves one-by-one, it became uncomfortable as those who had university affiliations reported them, as juxtaposed with those who did not. By not carefully scripting our introductory protocol, we had unintentionally reified a divide between ourselves and our community researchers, and created an educational hierarchy among our participants. We subsequently revised our introductions protocol so that all university affiliations were omitted. Instead, we talked about ourselves as individuals who had been drawn to this project based on a variety of factors about our identities.

\section{Lessons Learned This Study}


Subtle Factors Easily Overlooked. There are many subtle considerations that are less important in the classroom that become incredibly important in training community researchers. For example, during our first and second series of sessions, we distributed some readings that we drew from introductory research methodology textbooks regarding best practices in recruiting and interviewing. In a classroom setting, we would expect our students to complete these readings outside of class time, and come back prepared to discuss what they had read. While we initially saw value in giving community researchers some 'homework' reading related to the project, we noticed in our follow-up sessions that the reading materials we distributed in folders did not appear to have been reviewed. In retrospect, we realized that the expectation to read methodological literature, however introductory and light in nature, may have been unreasonable given that our community researchers had already volunteered their time to participate during the sessions.

Training Is Not Collaboration. We framed our sessions with community researchers as 'training sessions'. In part due to federal guidelines on research ethics, and the regulatory requirement that we provide 'ethics training' to community researchers, our initial sessions took a very didactic tone. There were university-affiliated researchers essentially lecturing on ethics and conduct of research. That training model was far from collaborative - it positioned the university-affiliated researchers as experts and the community researchers as passive recipients of that expertise. We observed in those moments that a power dynamic was developing - one participant even referred to an Auburn researcher as her "boss". We corrected that misunderstanding by emphasizing we were collaborators in this project, but that community researcher's comment likely indicates a larger problem with the structure of the 'training sessions'.

We have made efforts to correct this going forward. While our university IRB requires that we provide community researchers with training modules followed by multiplechoice quiz questions, we are working to make this a more humanizing and less didactic experience. Now, in the live session, we use scenarios for discussion - emphasizing that there might be multiple valid opinions on how to handle some situations - and save the multiple choice questions and correct responses for the take-home readings. We ask participants to look through these and self-check their understanding, but we do not quiz during our time together.

We have also moved from presenting our interview protocol as-is, to presenting it along with a worksheet asking community researchers to create their own. We now openly talk about why we had to make an interview protocol before talking with the community researchers, and ask them to adapt the questions. We have an open conversation about those items. While we are bound by regulation to stick to the same topics as our interview protocol, we positioned that protocol as a loose idea of the type of questions we might ask, rather than as a structured questionnaire. That element of our IRB protocol gives the flexibility for us to work with community researchers on creating their own protocol, so long as it is related to the same topics as the IRB-reviewed and researcher-created version.

Community Organization Goals and Researcher Goals. By their nature, universities (and university researchers) have different goals from community organizations. Such organizations are oriented toward advocacy, social change, organizing, and fundraising. Our work as university-affiliated researchers is necessarily oriented toward empirical research, trustworthy findings, and publishable results. Though several on our team would identify as scholar-activists (meaning we see our research as useful in creating social change 
toward equity and social justice), our employment at a university still dictates research priorities. This misalignment was visible in several ways.

In one instance, we noted that a community organization representative was working to collect contact information from community researchers at a training session. That was a natural part of the representative's typical work, but felt oddly out of place in the research setting. In another instance, a community organization representative talked openly about the need to challenge heteronormative or transmisogynistic ideas during interviews. While that goal is certainly appropriate for an LGBTQ community organization, it was not an appropriate goal in the conduct of research. (While the results might be useful in challenging problematic ideas, the research process itself was not the right place for such a challenge).

In instances where we noticed a difference in our goals, the solution was not typically simple. We were, in most cases, guests in spaces owned and used by LGBTQ community organizations. Attempts to 'correct' could easily reinforce an unequal power dynamic. We sought instead to gently suggest how we might align our goals. For example, we talked with the representative advocating for using the research interviews to challenge and discussed how that challenge would likely be more productive once the data were in, and talked about questions that such a challenge in the interviews could create about our findings.

Changing the Dynamic. We discuss above the problems with marginalization and power dynamics in a study like this one. How can researchers work to reduce the role of those power dynamics and work to create authentic community? We don't have a definite answer, but offer some suggestions based on our experience:

- Collaborate early and often with community members and organizations

- Build time for collaboration and multiple IRB submissions into the project timeline

- While training is required by regulations, actively work against creating a didactic environment

- Have open and frank conversations with community organizations about their goals, the researchers' goals, and how they might overlap and conflict

- Talk openly about which parts of the project have been researcher-driven, why, and how community involvement is honored in the project

- Explore strategies for creating stronger community affiliation among those involved with the project, such as plans for interaction outside the research context

\section{Conclusions}

We have described a number of the challenges to putting CBPR into action in our study. Having worked through many of these challenges, however, we feel strongly that the CBPR approach is worth the extra effort and worth the problems it creates. Many of the community researchers we worked with in Alabama and Mississippi expressed that this project is among the first times they have felt empowered to create change in their locale. Some expressed a long-standing desire to contribute to LGBTQ communities, but difficulties in finding ways to contribute. Moreover, we have had more success in reaching LGBTQ people of color in the South than almost any prior researchers. Community members have the ability to reach more deeply into the LGBTQ community, and several have interviewed people who are not 'out'. A university-affiliated researcher would likely be unable to reach those participants, so without the CBPR approach, those needs and experiences would go entirely undocumented. 
The CBPR approach has been, and continues to be, challenging, particularly given institutional and community constraints. However, it has also been extremely rewarding, both in the interactions with community researchers, and with the quality and depth of the data obtained. CBPR work takes a careful, humble, and thoughtful approach, but offers many advantages over traditional methods, particularly for understanding marginalized communities.

\section{Exercises and Discussion Questions}

1. What is the purpose of community-involved research methods? How are they different from more traditional research methods?

2. What populations and research questions are most appropriate for communityinvolved research and community-based participatory research? Why might some populations or questions be less appropriate for these methods?

3. In what ways are LGBTQ communities unique? What are some of the challenges of research with this population? How is this similar to and different from other traditionally marginalized populations?

4. Thinking about your own research interests, how might community-involved and community-based participatory research methods work with your research?

5. What are some of the challenges identified in this case study? How might those challenges be similar to or different from the challenges you might face in your own research work?

6. Create a plan for community-involved or community-based participatory research.

a. What are the key constituencies, organizations, or groups you might need to coordinate with or work alongside?

b. How will you build relationships in the community of interest?

c. What steps would you take to find an authentic research question based on community needs?

d. What types of community involvement do you believe would be appropriate to this population? 
e. How will you go about involving community members throughout the research process?

\section{Further Readings}

Hacker, K. (2013) Community-based participatory research. Thousand Oaks, CA: SAGE.

Johnson, L. R. (2017). Community-based qualitative research: Approaches for education and the social sciences. Thousand Oaks, CA: SAGE.

Paris, D., \& Winn, M. T. (2014). Humanizing research: Decolonializing qualitative inquiry with youth and communities. Thousand Oaks, CA: SAGE.

\section{References}

Austin, E. L., \& Irwin, J. A. (2010a). Age differences in the correlates of problematic alcohol use among southern lesbians. Journal of Studies on Alcohol and Drugs, 71(2), 295298.

Austin, E. L., \& Irwin, J. A. (2010b). Health behaviors and health care utilization of southern lesbians. Women's Health Issues, 20(3), 178-184.

Baunach, D. M., Burgess, E. O., \& Muse, C. S. (2010). Southern (dis)comfort: Sexual prejudice and contact with gay men and lesbians in the South. Sociological Spectrum, 30(1), 30-64.

Berg, R. C., \& Ross, M. W. (2014). The second closet: A qualitative study of HIV stigma among seropositive gay men in a Southern U.S. city. International Journal of Sexual Health, 26(3), 186-199.

Craig, S. L., \& Keane, G. (2014). The mental health of multiethnic lesbian and bisexual adolescent females: The role of self-efficacy, stress, and behavioral risks. Journal of Gay and Lesbian Mental Health, 18(3), 266-283.

Geter, A., Ricks, J. M., McGladrey, M., Crosby, R. A., Mena, L. A., \& Ottmar, J. M. (2016). Experiences of antihomosexual attitudes and young Black men who have sex with men in the South: A need for community-based interventions. LGBT Health, 3(3), 214-218.

Hacker, K. (2013) Community-based participatory research. Thousand Oaks, CA: SAGE. 
Johnson, L. R. (2017). Community-based qualitative research: Approaches for education and the social sciences. Thousand Oaks, CA: SAGE.

Human Rights Campaign. (n.d.). Human Rights Campaign unveils results of largest LGBT survey in Mississippi history. Human Rights Campaign Mississippi. Retrieved from http://hrc-assets.s3-website-us-east-1.amazonaws.com//files/assets/resources/ Mississippi_Messaging_7.14.pdf

MacCarthy, S., Mena, L., Chan, P. A., Rose, J., Simmons, D., Riggins, R., ... Nunn, A. (2015). Sexual network profiles and risk factors for STIs among African-American sexual minorities in Mississippi: A cross-sectional analysis. LGBT Health, 2(3), 276281.

Mallory, C., Flores, A., and Sears, B. (2016). LGBT in the South. The Williams Institute. Retrieved from http://williamsinstitute.law.ucla.edu/research/census-lgbtdemographics-studies/lgbt-in-the-south/.

Ozer, E. J. (2016). Youth-led participatory action research. In Jason, L. A. \& Glenwick, D. S. (Eds). (2016). Handbook of methodological approaches to community-based research: Qualitative, quantitative, and mixed methods. New York, NY: Oxford University Press.

Shelton, K., \& Delgado-Romero, E. A. (2011). Sexual orientation microaggressions: The experience of lesbian, gay, bisexual, and queer clients in psychotherapy. Journal of Counseling Psychology, 58(2), 210-221.

Slater, L. Z., Moneyham, L., Vance, D. E., Raper, J. L., Mugavero, M. J., \& Childs, G. (2015). The multiple stigma experience and quality of life in older gay men with HIV. Journal of the Association of Nurses in AIDS Care, 26(1), 24-35.

Whyte, W. F. (1989). Advancing scientific knowledge through participatory action research. Sociological Forum, 4(3), 367-385.

Williams Institute. (2016). LGBT people in Alabama. Census and LGBT Demographic Studies. Retrieved June 16, 2016 from http://williamsinstitute.law.ucla.edu/wpcontent/uploads/Alabama-Fact-Sheet.pdf. 